\title{
El rol de la cultura en la diplomacia iberoamericana. Análisis de las Declaraciones de las Cumbres Iberoamericanas de Jefes de Estado y de Gobierno (1991-2018)
}

\section{The role of culture in Ibero-American Diplomacy. Analysis of the Declarations of the Ibero-American Summits of Heads of State and Government (1991-2018)}

\author{
Ian Henríquez Herrera ${ }^{1}$ \\ Universidad Finis Terrae (Chile) \\ ORCID: https://orcid.org/0000-0001-9905-0750
}

\author{
Rut Sánchez Espiga ${ }^{2}$ \\ Universidad Francisco Vitoria (España) \\ ORCID: https://orcid.org/0000-0001-5894-5013
}

Recibido: 20-04-2021

Aceptado: 18-05-2021

\section{Resumen}

El presente trabajo indaga sobre el papel de la cultura en la diplomacia. Para la diplomacia es indispensable el diálogo, el cual, para ser fructífero, no puede prescindir de la cultura. En el caso de Iberoamérica, la cultura se muestra como una clara oportunidad de vinculación estable y paz duradera. ¿Cuál ha sido el rol

\footnotetext{
1' (ihenriquez@uft.cl). Doctor en Derecho y Magíster en Investigación Jurídica por la Universidad de los Andes. Magíster en Derecho Privado y Licenciado en Ciencias Jurídicas y Sociales por la Universidad de Chile. Director del Centro Wojtyla para el Estudio de la Persona de la Universidad Finis Terrae. Profesor titular de la Facultad de Derecho de la Universidad Finis Terrae. Algunas publicaciones recientes: "Las personas en situación de prostitución: ¿disposición sobre el propio cuerpo o cosificación de la subjetividad humana?", en Cuadernos de Bioética de la Asociación Española de Bioética, $\mathrm{n}^{\circ}$ 103, 2020, en coautoría con Pamela Cajales; "Los efectos relativo y absoluto del contrato de transacción", en Vniversitas (Colombia), n 69, 2020; “¿Tiene sentido que los juristas hablen de «razas»?, en Revista Crítica de Derecho Privado (Uruguay), n¹3, 2016; "Lettres de Jacques, Räissa et Gabriela Mistral”, en Cahiers Jacques Maritain, № 74, en coautoría con Alejandro Serani.

2 (rutsanchezespiga@gmail.com). Periodista graduada en Relaciones Internacionales por la Universidad Francisco Vitoria (España).
} 
que se le ha asignado a la cultura en las Cumbres Iberoamericanas de Jefes de Estado y Gobierno? Para contestar dicha pregunta, el método de investigación que hemos seguido ha sido triple: se ha hecho uso de fuentes bibliográficas, entrevistas a expertos y análisis de texto de las Declaraciones fruto de las referidas Cumbres Iberoamericanas.

Palabras-clave: Cultura, paz, diplomacia cultural, Iberoamérica.

\begin{abstract}
This work discusses the role of culture in diplomacy. Dialogue is indispensable for diplomacy, which, in order to be fruitful, cannot do without culture. In the case of Ibero-America, culture is shown as a clear opportunity for stable bonding and lasting peace. What has been the role assigned to culture at the Ibero-American Summits of Heads of State and Government? To answer this question, the research method we have followed has been threefold: bibliographic sources, expert interviews and text analysis of the Declarations resulting from the aforementioned Ibero-American Summits have been used.
\end{abstract}

Keywords: Culture, Peace, Cultural Diplomacy, Ibero-America

\title{
1. Introducción
}

El siguiente trabajo indaga sobre el papel de la cultura en la diplomacia. Presupone que el objeto de esta última es la búsqueda de la paz ${ }^{3}$. Nuestra intuición primera, es que una cultura compartida es facilitadora en dicha búsqueda. Si ello es así, como buenas razones parecen mostrar, la centralidad de la primera -la cultura- en la segunda - la diplomacia- debería ocupar nuestra atención de una manera preferente. Para la diplomacia es indispensable el diálogo, y en este diálogo -etimológicamente, pasar a través del logos - no se puede prescindir de la cultura, puesto que, simplemente para comenzar a conversar, hace falta encontrar un punto en común, una lengua que pueda ser interpretada

\footnotetext{
${ }^{3}$ La diplomacia es, de acuerdo con Vilariño, "aquella actividad ejecutora de la política exterior de un sujeto de derecho internacional, llevada a cabo por órganos y personas debidamente representativos del mismo, ante otro u otros sujetos de derecho internacional para, por medio de la negociación, alcanzar, mantener o fortalecer transaccionalmente la paz; ha de tener como finalidad última hacer posible, con tales medios, la construcción o existencia de una comunidad internacional justa que, a través de la cooperación, permita el pleno desarrollo de los pueblos". Eduardo Vilariño Pintos, Curso de Derecho Diplomático y Consular. Parte General y textos codificados, Editorial Tecnos, Madrid, 1987. También Rafael Calduch mantiene esta afirmación al explicar que "en toda relación diplomática, y sea cual sea el objetivo inmediato de la acción exterior por el que dicha relación se ha entablado, la finalidad última que justifica su existencia y le da pleno significado es la de alcanzar o mantener unas relaciones internacionales pacíficas". Rafael Calduch, Dinámica de la Sociedad Internacional, Edit. CEURA. Madrid, 1993.
} 
por ambos interlocutores. Si bien cultura e identidades han sido fuente de conflicto en numerosas ocasiones, solo a través de las mismas podemos llegar al establecimiento de una paz duradera, puesto que en ellas se encuentran expresiones profundas del ser humano. La pérdida de la identidad que caracteriza a las sociedades occidentales post modernas, con la consiguiente liquidez de valores, mina la capacidad de generar vínculos estables y permanentes. Fundar las relaciones en conceptos frágiles o volátiles conduce a los Estados a una política exterior cortoplacista y efímera. Este trabajo busca ser una aportación más en favor de la centralización de la cultura en la diplomacia.

En el caso de Iberoamérica, la cultura se muestra como una clara oportunidad de vinculación estable y paz duradera, que no debe ser olvidada. ¿Cuál ha sido el rol que se le ha asignado a la cultura en las Cumbres Iberoamericanas de Jefes de Estado y Gobierno? Para contestar dicha pregunta, el método de investigación que hemos seguido ha sido triple: se ha hecho uso de fuentes bibliográficas, fuentes personales por medio de la entrevista a cinco expertos en distintas áreas, y análisis de texto de las Declaraciones fruto de las referidas Cumbres Iberoamericanas.

\section{Diplomacia cultural iberoamericana}

Para poder llevar a cabo un diálogo, un proceso de integración verdadero en Iberoamérica, debe abandonarse la visión de verticalidad en las relaciones entre países. Como Fred Dallmayr ${ }^{4}$ indica, la memoria del colonialismo ha llevado a una tendencia a ver la cultura occidental como sinónimo de arrogancia cultural, por lo que, para poder avanzar, es necesario "delinear las estructuras y precondiciones del diálogo civilizacional"5. Quedarse anclado en la historia supone la imposibilidad de establecer un diálogo que no incluya la superioridad moral de unos sobre otros. Es por ello que el historiador peruano Jorge Bustos exclama su célebre frase: "No somos ni vencedores ni vencidos, somos los descendientes de los vencedores y vencidos" ". Se trata de reconocer que nos encontramos en una simbiosis en la que ni España se entendería sin Latinoamérica, ni Latinoamérica puede desvincularse de parte de su identidad, "virar la relación de dependencia morbosa con respecto del imperio español para transformarla en una relación nutritiva con respecto al presente"

\footnotetext{
${ }^{4}$ Fred Dallmayr, Dialogue among Civilizations: Some Exemplary Voices. Palgrave. Nueva York, 2002. Consultado en Isaac Caro; Isabel Rodríguez, "El enfoque del diálogo civilizacional desde América Latina”, Revista de Relaciones Internacionales, Estrategia y Seguridad, vol. 11, núm. 1, Bogotá, Colombia, 2016, p. 155.

5 Fred Dallmayr, "Dialogue among Civilizations: Some Exemplary Voices", ibid.

${ }^{6}$ Alfredo Figueiras Fernández-Ojanguren, Ciudades Españolas en América, Ediciones Lacre, 2017.

${ }^{7}$ María Elvira Roca Barea, "Estados Unidos, España y el Mundo hispánico”, 20 de diciembre de 2017. Disponible en: https:/www.youtube.com/watch?v=njI81X4E0IQ
}

Araucaria. Revista Iberoamericana de Filosofí, Política, Humanidades y Relaciones Internacionales, año $23, \mathrm{n}^{\circ} 47$. Segundo cuatrimestre de 2021. Pp. 507-527. ISSN 1575-6823 e-ISSN 2340-2199 https://dx.doi.org/10.12795/araucaria.2021.i47.22 
Para llegar hasta el estatus actual de las relaciones internacionales en Latinoamérica, previamente ha habido una etapa de tendencia nacionalista, predominante durante el siglo XIX y parte del siglo $\mathrm{XX}^{8}$, donde la inestabilidad política, económica y social requería años de formación de la identidad nacional y delimitación de fronteras. "Lo que brota en Ibero-América, ya no es la planta europea intacta, sino una tercera dimensión de sangre y cultura, enriquecida con aportes dispares y orientada a nuevos y no soñados destinos", escribe Jaime Eyzaguirre?

Actualmente, la "relación de España con América Latina es económicamente importante, políticamente compleja y culturalmente próxima" ${ }^{10}$. El ministerio de Asuntos Exteriores Español ${ }^{11}$ hace referencia a Iberoamérica como una región con la que generar un nuevo vínculo renovado con base en intereses comunes, con el fin de afianzar las relaciones políticas y el diálogo existente. Como señala Carlos Malamud, no debe olvidarse que la realidad de América Latina no solo es diversa, sino que también fragmentada, marcada por los distintos gobiernos y cambios políticos. Es por ello que, afirmada la pertenencia de España a la región Iberoamericana, la política exterior debe poner gran empeño en las relaciones bilaterales por medio de una "política latinoamericana" en la que se marque bien cada relación con los distintos países, reconociendo así de forma individual la importancia de cada país para España, especialmente en el ámbito cultural.

La cuestión iberoamericana, sobre todo, lleva a España a dar un papel relevante a Latinoamérica en su política exterior, al estar unida por algo más allá de los cambiantes gobiernos, "con independencia de la mayor o menor afinidad política" 12 , conservando un vínculo que debiera perdurar más allá de la ideología de sus representantes.

La "mundialización" que irrumpe en el siglo XXI provoca que las culturas adopten un rol todavía más determinante debido al "desdibujamiento" de los Estados en su concepción tradicional, "con unas fronteras delimitadas en función de un territorio y una cultura nacional como factor de identidad" 13 . Ahora Iberoamérica busca un reconocimiento como bloque regional a nivel mundial, aunque en la articulación de este pluralismo es importante no dejar de incidir en la base cultural compartida.

España ha tenido un gran peso en la construcción del espacio

8 Edmundo Aníbal Heredia, Relaciones internacionales latinoamericanas: historiografias $y$ teorías, Estudios Ibero-Americanos, PUCRS, v. XXXIV, n. 1, 2008, p.11.

9 Jaime Eyzaguirre, Hispanoamerica del dolor, Editorial Universitaria, 1968.

10 https://www.eleconomista.es/especial-america/noticias/9170918/05/18/El-compromiso-deEspana-con-America-Latina.html

${ }^{11} \mathrm{http}$ ///www.exteriores.gob.es/Portal/es/PoliticaExteriorCooperacion/Iberoamerica/Paginas/ EspEnIberoamerica.aspx

12 http://www.exteriores.gob.es/Portal/es/PoliticaExteriorCooperacion/Iberoamerica/Paginas/ EspEnIberoamerica.aspx

13 María del Rosario Fernández Santamaría, "Cultura y mundialización en el contexto iberoamericano", Pensar Iberoamérica, Revista de Cultura, Organización de Estados Iberoamericanos. 
Iberoamericano, siendo el país del que parte la propuesta. Un exceso de la participación de España en el proceso de desarrollo de Iberoamérica genera desconfianza acerca del rol que pretende adoptar. Es por ello que la presencia de los países "lusófonos" puede contribuir a distribuir las fuerzas y garantizar una "iberoamericanización de las cumbres" que facilite la disposición del resto de países iberoamericanos ${ }^{14}$. Evitar la "españolización" del proyecto para verdaderamente crear relaciones horizontales en un nuevo espectro internacional.

Establecido este marco, es conveniente analizar la particularidad del fenómeno Hispanoamericano y con ello comprender el potencial directo de paz duradera en Iberoamérica. Los países lusófonos no quedan fuera de este análisis, que en su caso ha de hacerse con el caveat de la ligera diferencia en el uso de la lengua, elemento relevante en la configuración de la cultura.

\subsection{La particularidad deIberoamérica: el fenómeno Hispanoamericano y los países lusófonos}

El concepto de "Iberoamérica" es fruto de una historia compartida, de unas raíces y un patrimonio cultural conjunto pero rico en sus diversas expresiones. Se trata de "una de las comunidades de naciones más naturales, verdaderas y espontáneas del planeta", por su carácter preexistente a cualquier institución contemporánea ${ }^{15}$. Pese a la vastedad de países que conforman Iberoamérica, con complejos intereses e ideologías, están relacionados por un elemento más fuerte que los conecta: la cultura.

En toda cultura se pueden distinguir cinco componentes: los símbolos, el lenguaje, los valores, las normas y los objetos materiales ${ }^{16}$. Los símbolos, los valores, las normas remiten al factor religioso, en tanto que modelan una cosmovisión, una forma de ver, de comprender e interpretar el mundo. El caso del fenómeno hispanoamericano es notable y probablemente único, puesto que hay dos elementos claros que configuran un nexo básico entre múltiples naciones: el idioma español y la religión católica como base cultural. La existencia de una comunidad que comparta estas características es una oportunidad de paz invaluable, puesto que no elimina la independencia de los Estados en sus particularidades, pero los vincula entre sí de un modo fuerte y perdurable. Se trata de una región cultural de una inmensa extensión territorial y con una comunidad de hispanohablantes cada vez más dispersa

\footnotetext{
${ }^{14}$ http://www.realinstitutoelcano.org/wps/wcm/connect/88e57f8f-e83e-49e4-9817-76551a6ble8a/ DT11-2017-FerreiraGomes-Espana-Portugal-espacio-iberoamerican-coincidencia-prioridades.pdf? MOD=AJPERES\&cacheid=1509987679510

15 Enrique Iglesias, "El español en iberoamérica: lengua e identidad", TELOS 71: El valor económico del español en la Sociedad Global, Agenda, $\mathrm{n}^{\mathrm{o}} 71$.

16 John Macionis, Ken Plummer, Sociología, Prentice Hall, 1999, p.108.
} 
por todo el globo. De hecho, el fenómeno migratorio latino hacia USA es incluso meritorio de un análisis particularizado, que, por cierto, excede el marco de este estudio, pero que reditúa la importancia de la unidad cultural hispanoamericana.

Portugal y Brasil comparten con Hispanoamérica una gran afinidad cultural, con lazos históricos que los unen y la presencia geográfica a ambos lados del Atlántico. También comparten la unidad de la base religiosa, y, desde el punto de vista de la comprensión entre idiomas, lingüística ${ }^{17}$.

\subsection{Extensión territorial}

La extensión territorial de Iberoamérica es notoriamente vasta. ¿Qué otra región cultural puede presumir de tal amplitud, de encontrarse en dos continentes? Poder generar un vínculo duradero en toda la región Iberoamericana supondría establecer relaciones de paz en 22 países, 19 de América Latina (Argentina, Bolivia, Brasil, Colombia, Costa Rica, Cuba, Chile, República Dominicana, Ecuador, El Salvador, Guatemala, Honduras, México, Nicaragua, Panamá, Paraguay, Perú, Uruguay y Venezuela), y 3 de la península Ibérica, agrupando España, Andorra y Portugal. Unido a esto hay elementos geoestratégicos: la posición de Portugal y España como puente en Europa y la influencia de Latinoamérica en Estados Unidos. No se debe olvidar la importancia de la demografía, ya que existe una inmensa comunidad de hispanohablantes inserida en el país que más presume de "poder blando": Estados Unidos.

La vasta extensión territorial de Latinoamérica podría considerarse como una debilidad en el desarrollo. Las distancias son enormes, Brasil es tan grande como los Estados Unidos continentales y Argentina es tan grande como la India, aunque mucho menos poblada. La comunicación entre la capital y las zonas rurales en los países grandes es muy complicada y existen barreras naturales como la cordillera de los Andes y la cuenca del Amazonas. La globalización está desmontando la mayor parte de estas teorías.

La existencia de una región como la Iberoamericana brinda una oportunidad privilegiada para la conducción de relaciones pacíficas en una gran parte del planeta, lo cual, a su vez, puede incidir positivamente a nivel global, al significar un punto de gravitación y de estabilidad. Estos aspectos hay que considerarlos y ponderarlos con suficiente atención.

\footnotetext{
${ }^{17}$ Nancy Elena Ferreira Gomes, España y Portugal en el espacio Iberoamericano: coincidencia en las prioridades de política externa, Real Instituto Elcano, 2017.
} 


\subsection{La unidad de la lengua}

El lenguaje constituye "el mecanismo más importante de reproducción cultural"18 de una sociedad. Se trata del sistema de símbolos por medio del cual los miembros de una sociedad se comunican y preservan el conocimiento, una de las herramientas más importantes para conservar la propia cultura. Para Iberoamérica el idioma es uno de los valores culturales compartidos más relevantes.

A través del lenguaje se observa y se explica el mundo, por lo que configura la realidad. La hipótesis de Sapir-Whorf mantiene que "las personas perciben el mundo de una u otra forma dependiendo de la lengua que hablan"19. Un anglosajón, en su estudio del español, tendrá gran dificultad para comprender la diferencia entre "ser" y "estar" que establecen los hispanohablantes. Es célebre y conocido el verso de Benedetti, del poema "Ser y estar": "Oh marine / oh boy / una de tus dificultades consiste en que no sabes / distinguir el ser del estar / para ti todo es to be / así que probemos a aclarar las cosas".

Este es solo un pequeño ejemplo de por qué el idioma español, en sus particularidades, actúa como "fuerza de amor y de cohesión espiritual" Cuanto menores interferencias haya en la simbología de las cosas, cuanto mayor sea la similitud del entendimiento, mayor será la facilidad de creación del vínculo. Estamos unidos por la sutileza en la comprensión de los tiempos verbales, el fácil tránsito del sustantivo al verbo, la notoria extensión de sustantivos comunes que delimitan la naturaleza, etc.

El rol de la lengua ocupa un lugar fundamental en la unidad cultural. No es casualidad que gentilicios y lengua se llamen de un mismo modo. De alguna manera, cuya comprensión misteriosamente nos excede, somos lo que hablamos. Por algo Heidegger, en su célebre carta sobre el humanismo, acuñó la famosa alusión al lenguaje como "la casa del ser".

La riqueza de la lengua castellana es un bien preciadísimo, y el siguiente texto de Neruda, "Las palabras", por su expresividad, amerita su transcripción:

Todo lo que usted quiera, sí señor, pero son las palabras las que cantan, las que suben y bajan... Me prosterno ante ellas... Las amo, las adhiero, las persigo, las muerdo, las derrito... Amo tanto las palabras... Las inesperadas... Las que glotonamente se esperan, se escuchan, hasta que de pronto caen... Vocablos amados... Brillan como piedras de colores, saltan como platinados peces, son espuma, hilo, metal, rocío... Persigo algunas palabras... Son tan hermosas que las quiero poner todas en mi poema... Las agarro al vuelo, cuando van

\footnotetext{
${ }_{18}$ John Macionis, Ken Plummer, Sociología, Prentice Hall, 1999, p. 109,111.

${ }^{19}$ Ídem.

${ }^{20}$ Palabras del expresidente Mexicano Miguel Alemán Valdés en su propuesta de celebración del primer «congreso de Academias de habla española» (1950). Consultado en https://www.asale.org/ sites/default/files/Folleto_de_ASALE.pdf [02/05/2020]
} 
zumbando, y las atrapo, las limpio, las pelo, me preparo frente al plato, las siento cristalinas, vibrantes, ebúrneas, vegetales, aceitosas, como frutas, como algas, como ágatas, como aceitunas... Y entonces las revuelvo, las agito, me las bebo, me las zampo, las trituro, las emperejilo, las liberto... Las dejo como estalactitas en mi poema, como pedacitos de madera bruñida, como carbón, como restos de naufragio, regalos de la ola... Todo está en la palabra... Una idea entera se cambia porque una palabra se trasladó de sitio, o porque otra se sentó como una reinita adentro de una frase que no la esperaba y que le obedeció... Tienen sombra, transparencia, peso, plumas, pelos, tienen de todo lo que se les fue agregando de tanto rodar por el río, de tanto transmigrar de patria, de tanto ser raíces... Son antiquísimas y recientísimas... Viven en el féretro escondido y en la flor apenas comenzada... Qué buen idioma el mío, qué buena lengua heredamos de los conquistadores torvos... Estos andaban a zancadas por las tremendas cordilleras, por las Américas encrespadas, buscando patatas, butifarras, frijolitos, tabaco negro, oro, maíz, huevos fritos, con aquel apetito voraz que nunca más se ha visto en el mundo... Todo se lo tragaban, con religiones, pirámides, tribus, idolatrías iguales a las que ellos traían en sus grandes bolsas... Por donde pasaban quedaba arrasada la tierra... Pero a los bárbaros se les caían de las botas, de las barbas, de los yelmos, de las herraduras, como piedrecitas, las palabras luminosas que se quedaron aquí resplandecientes... el idioma. Salimos perdiendo... Salimos ganando... Se llevaron el oro y nos dejaron el oro... Se lo llevaron todo y nos dejaron todo... Nos dejaron las palabras ${ }^{21}$.

Así, los hispanohablantes configuran una "comunidad idiomática". El español, en sus diversos modelos lingüísticos, genera un "inalienable motivo de unión interamericana y de relación con España"22. Bajo el uso de una misma lengua, las particularidades de cada lugar protegen y son seña de la cultura propia: no desearíamos una homogenización cultural. Lo que se está buscando es una integración cultural, y el idioma compartido favorece esta integración de un modo extraordinario. La unidad de la lengua nos comunica -hace comunión, nos transforma en comunidad- y las diferencias de usos locales, si sabemos integrarlas, nos enriquece. Para poder establecer vínculos duraderos, el idioma español debe verse bajo la mirada de una "lengua compartida y en constante construcción y recreación por todos los hispanohablantes a ambos lados del Atlántico"23. Cuanto mayor sea el esfuerzo por recoger y comprender la diversidad de expresiones de la lengua, mayor será el acercamiento entre los Estados.

${ }^{21}$ Disponible en Revista Altazor, Revista Electrónica de Literatura, año 3, enero 2021. https://www. revistaaltazor.cl/pablo-neruda-las-palabras/.

22 Juan Antonio Frago Gracia, Entre América y España, el vínculo de la lengua, Boletín de Filología vol.47 no.2 Santiago dic. 2012.

${ }^{23} \mathrm{http}$ ://www.realinstitutoelcano.org/wps/portal/rielcano_es/contenido?WCM_GLOBAL CONTEXT=/elcano/elcano_es/zonas_es/politicaexteriorespanola/ari12-2019-malamud-espanaentre-america-latina-e-iberoamerica 
Esta lengua viva y cambiante se recoge actualmente en las 22 Academias de la Lengua que abarcan todas las variantes del castellano. La ASALE, la Asociación de Academias de la Lengua Española, fue galardonada con el Premio Príncipe de Asturias de la Concordia 2000, por su labor en favor de la lengua como vehículo de entendimiento y de concordia entre los pueblos ${ }^{24}$. También entre los mecanismos que ayudan a articular la cultura iberoamericana en términos de lenguaje se encuentra la labor del Instituto Cervantes, que promueve el "patrimonio lingüístico y cultural" común de la comunidad hispanohablante 25 . "La maravilla de la semejanza, el toque de gracia caído sobre esos veinte pueblos, y de donde parten todos los bienes actuales y venideros de la unidad, hay que adjudicarla a la lengua", escribe Gabriela Mistral en Fiesta de la lengua española ${ }^{26}$. Las diferentes variaciones del español lo enriquecen y favorecen la unicidad de cada país hispanoamericano.

La importancia de la literatura es clave en la relación de intercambio hispanoamericano. No solo se comparte la variedad de la propia lengua, sino también el imaginario, los símbolos diversos que pueda esconder. El boom de la literatura latinoamericana en los años 60 llena a España de un nuevo paisaje, de nuevas visiones sobre el mundo y las cosas, de una perspectiva mágica de la realidad. Curioso es el hecho de que la diplomacia en América Latina haya estado repleta de intelectuales, escritores, poetas, que han favorecido el prestigio cultural de su propio país en el exterior. Hasta el momento, son seis los premios Nobel de Literatura concedidos a escritores latinoamericanos. De ellos, cuatro han sido a su vez figuras importantes dentro del mundo de la diplomacia: Gabriela Mistral (1945, Chile), Miguel Ángel Asturias (1967, Guatemala), Pablo Neruda (1971, Chile), Octavio Paz (1990, México). Comúnmente se ha justificado este fenómeno con el hecho de que, al ser representantes de la cultura en sus países, eran útiles a la hora de proyectar una buena imagen ${ }^{27}$. El razonamiento podría ir más allá de la mera apariencia: estos personajes eran capaces de comprender en mayor medida el valor de la propia cultura y de hacerla presente en la política exterior.

Desde el punto de vista de la propia conservación de la cultura, la literatura es fundamental. Hay una "república de las letras compartida" constante en los últimos dos siglos ${ }^{28}$. "Los descubridores de García Márquez o de Vargas

\footnotetext{
${ }^{24} \mathrm{https}$ //Www.asale.org/sites/default/files/Folleto_de_ASALE.pdf

$25 \mathrm{https}$ ://www.cervantes.es/sobre instituto_cervantes/informacion.htm

${ }^{26}$ Juan Antonio Frago Gracia, Entre América y España, el vínculo de la lengua, Boletín de Filología vol.47 no.2 Santiago dic. 2012.

27 Carlos Marichalar, Algunas reflexiones sobre la historia de los intelectuales/diplomáticos latinoamericanos en los siglos XIX y XX, Revista de Historia de América, Instituto Panamericano de Geografía e Historia, núm. 156, 2019.

${ }_{28}$ Manuel Lucena Giraldo, historiador doctorado en Historia de América, investigador del CSIC, profesor y experto en educación y cultura, en entrevista concedida a Rut Sánchez Espiga el día 06/05/2020 vía video llamada desde Madrid.
} 
Llosa son editoriales españolas. Y antes ya se había producido a la inversa, todos los intelectuales del exilio español después de la Guerra Civil publican fundamentalmente en México y en Argentina, y sin esas publicaciones hubiera habido muchísimas obras de intelectuales, escritores, ensayistas españoles que se hubieran perdido", afirma Jose María González Ochoa ${ }^{29}$. Esto demuestra claramente que en el intercambio hay un motor de desarrollo e incluso de puesta en valor de riquezas no valoradas por el propio país. Los flujos de escritores e intelectuales Iberoamericanos son uno de los mejores ejemplos de una "comunidad cultural viva" 30 .

De esta unidad idiomática no forman parte, como es obvio, Brasil y Portugal. La identidad lingüística de Iberoamérica es doble: de ella forman parte el español y el portugués. Esto representa un obstáculo en la comunicación que los hispanohablantes no tienen. Aun así, el "hecho de la inteligibilidad práctica" ${ }^{31}$ entre ambos lenguajes explica la cohesión de la comunidad, sin perjuicio de otros elementos culturales que las vinculan. En el caso concreto de estos dos idiomas, son "las dos únicas grandes lenguas internacionales, en términos cuantitativos (habladas cada una de ellas por más de cien millones de personas), que son al mismo tiempo, y en líneas generales, recíprocamente comprensibles" ${ }^{32}$. Un ejemplo particular de esta inteligibilidad es el llevado a cabo por el poeta chileno Nicanor Parra, Premio Cervantes, y cuya obra ha tenido gran importancia en las letras hispanoamericanas. Citando a Pessoa, mezcla español y portugués en un mismo escrito sin que esto presente dificultad para el lector ${ }^{33}$.

No conviene olvidar, con relación a la unidad de la lengua, la cuestión indígena. De acuerdo con la Comisión Económica para América Latina y el Caribe, un $60 \%$ de la población originaria habla idioma vernáculo y castellano $^{34}$. Es evidente que la riqueza cultural de América Latina, resultado en sí de un "intenso proceso intercultural donde han convergido y se han fusionado naciones, lenguas, religiones y saberes" ${ }^{35}$. Encontramos variedades idiomáticas como el quechua en Chile, Perú, Bolivia y Ecuador, el aymara en

\footnotetext{
${ }^{29}$ José María Fernández Ochoa, historiador experto en la época de la Conquista y coordinador de proyectos para SM España y América Latina, en entrevista concedida a Rut Sánchez Espiga el día 27/04/2020 vía Skype desde Madrid, archivada en soporte electrónico.

30 José María Fernández Ochoa, en entrevista ya citada.

${ }^{31}$ Enrique Iglesias, "El español en Iberoamérica: lengua e identidad", TELOS 71: El valor económico del español en la Sociedad Global, Agenda, $n^{\circ} 71$.

${ }^{32}$ Ídem.

${ }^{33}$ Nicanor Parra, Ridiculo, ¿verdad? Discursos de sobremesa, Ediciones Universidad Diego Portales, Santiago de Chile, 2006, p. 277.

${ }^{34} \mathrm{https}: / /$ celade.cepal.org/redatam/PRYESP/SISPPI/Webhelp/porcentaje_de_indigenas_habla_ idioma_indigena_y_castellano.htm

${ }_{35}$ Edgar Montiē, Intervención en el Encuentro andino sobre Diplomacia Cultural. Seminario convocado por el Ministerio de Relaciones Exteriores de Colombia y la UNESCO (Bogota 27 y 28 septiembre 2007).
}

Araucaria. Revista Iberoamericana de Filosofia, Politica, Humanidades y Relaciones Internacionales, año $23, \mathrm{n}^{\circ} 47$. Segundo cuatrimestre de 2021. Pp. 507-527. ISSN 1575-6823 e-ISSN 2340-2199 https://dx.doi.org/10.12795/araucaria.2021.i47.22 
Bolivia y Chile, el guaraní en Paraguay, el mapudungún en Chile y Argentina, el Rapa Nui y el yámana en Chile, el nahualt en México y Guatemala, etc. Para poder llevar a cabo una verdadera integración cultural es necesario que los estados latinoamericanos pongan en valor sus particularidades y que estas sean acogidas por el conjunto de Iberoamérica, puesto que, igual que se debe huir de la política del no involucramiento, también es importante defender la cultura del Kulturkampf las "cruzadas culturales y la homogeneización opresiva"36.

\subsection{La unidad de la base religiosa}

La religión puede definirse como un segundo lenguaje, puesto que también reproduce la cultura y genera un código de símbolos compartidos. Por medio de la religión se establece un sistema de valores, una unidad ética, que dialoga acerca de la razón del ser. Las creencias permiten hacer afirmaciones descriptivas acerca del mundo, guiar el modo en que debemos comportarnos y definir los motivos de la propia existencia (MacIntyre, 1984) ${ }^{37}$. No se trata de una cuestión dogmática o fideísta: es una forma de ver el mundo y de comprender las cosas. Señala Posner:

El área de estudio de la antropología cultural es la cultura mental de una sociedad, en la medida en que se manifiesta en su civilización, esto es, su mentalidad. La mentalidad de una sociedad consiste en artefactos mentales (esto es, ideas y valores) y de convenciones que gobiernan sus usos y expresiones (...) Ejemplos de artefactos mentales religiosos son los santos católicos y sus emblemas, la clasificación de pecados con su correspondiente terminología ('pecado mortal', 'pecado venial', etc.), y los códigos gestuales de los sacerdotes ${ }^{38}$.

La cuestión religiosa muestra el modo en que la cultura trasciende la realidad presente ${ }^{39}$ y es capaz de abordar las problemáticas transversales a la historia del hombre, manifestando con ella una vocación de permanencia.

Pese a su notoria importancia en la identidad de los pueblos, no siempre ha sido debidamente tenida en cuenta en los análisis de política nacional o internacional:

La religión como fenómeno cultural y de identidad es un elemento de suma importancia en el análisis de las sociedades, aunque podemos decir que no suele ser con mucha frecuencia uno de los más atendidos, dada la complejidad y la diversidad que entraña su amplia cobertura. En tiempos recientes es traído a

\footnotetext{
${ }^{36}$ Zygmunt Bauman, La cultura en el mundo de la modernidad líquida, Polity Press, 2011, p.75.

37 James M. Nelson, Psychology, religion and spirituality, Springer Science \& Business Media, 2009.

${ }^{38}$ R. Posner, "Basic tasks of cultural semiotics", en: G. Withalm \& J. Wallmannsberger (Eds.). Signs of power - power of signs. Essays in Honor of Jeff Bernard. Vienna: INST.p. 10 (pp.56-89).

39 Zygmunt Bauman, “La cultura en el mundo de la modernidad líquida”, Polity Press, 2011, p.75.
} 
la superficie por el impacto que llega a tener su influencia en decisiones de tipo político, social, cultural e internacional ${ }^{40}$.

La religión católica ha moldeado las sociedades Iberoamericanas, pasando por sus ritos y tradiciones, sus relaciones e incluso sus palabras. Podemos ir desde la celebración del día de Todos los Santos a la idea de monogamia. Ya José Martí, en su conocido ensayo "Nuestra América", decía con su característica preclara inteligencia: "Con los pies en el rosario, la cabeza blanca y el cuerpo pinto de indio y criollo, venimos, denodados, al mundo de las naciones. Con el estandarte de la Virgen salimos a la conquista de la libertad"41. En efecto:

\begin{abstract}
América Latina es considerada una región eminentemente católica. Ello incluye básicamente a la parte continental y al Caribe hispano y francófono, pero también hay que considerar que, aunque minoritaria en algunos lugares, la Iglesia Católica está radicada también en el resto de las Antillas. Comúnmente se acepta el cálculo de que, al iniciarse el siglo XXI, en esta área geográfica residirá la mayor población católica del mundo. La Iglesia Católica es la institución eclesiástica más antiguamente establecida, hegemónica por largo tiempo, con mayores posibilidades de influencia en el ámbito social y estrechamente vinculada con los sistemas sociopolíticos e ideológicos establecidos en la mayoría de los países $^{42}$.
\end{abstract}

Esta clara unidad de la base religiosa no obsta al fenómeno contemporáneo de la "sociedad líquida", más bien lo permite. La religión subyace en la base de la cultura iberoamericana. De hecho, "en la actualidad la revalorización del legado indígena ocurre paralelamente a una nueva interpretación de ciertos rasgos centrales de la colonia española, entre los que se halla de manera preferente la religiosidad popular del periodo barroco en los siglos XVII y XVIII"43.

Por el lado de Latinoamérica, además, hay una tradición religiosa indígena que también ha influido en la visión del mundo y las cosas. Previa a la llegada de los misioneros católicos, los pueblos originarios practicaban diversas religiones politeístas, como la azteca, inca, maya, guaraní y mapuche, entre otras ${ }^{44}$. Entre

${ }^{40}$ Yubeira Zerba De Krispis, "Un acercamiento al fenómeno religioso en América Latina a la luz de la crítica cultural”, en Sapienza Organizacional, vol. 5, núm. 9, 2018, disponible en https://www. redalyc.org/jatsRepo/5530/553056570011/html/index.html

41 Publicado originalmente en La Revista Ilustrada de Nueva York, el 10 de enero de 1891. Disponible en: http://bibliotecavirtual.clacso.org.ar/ar/libros/osal/osal27/14Marti.pdf.

42 Jorge Ramírez Calzadilla, "La religiosidad americana y caribeña. Un elemento de la identidad cultural”, en América Latina y El Caribe. Realidades Sociopolíticas e Identidad Cultural, Ediciones Heinrich Böll, El Salvador, 2002, pp. 215-236. Disponible en http://biblioteca.clacso.edu.ar/ar/libros/ cuba/cips/caudales05/Caudales/ARTICULOS/ArticulosPDF/0915R072.pdf.

43 H.C.F. Mansilla, "La influencia de elementos religiosos sobre la cultura política en América Latina”, en Fragmentos de Filosofia, no 10 (2012), p. 92. (pp. 91-115).

44 Idelfonso Murillo Murillo, "La religión antes y después de las Independencias. ¿Fuente de unidad o de conflicto?”, Escritos, Vol. 19, N. 42, pp. 053-077, Medellín, Colombia, enero-junio 2011. http://www.scielo.org.co/pdf/esupb/v19n42/v19n42a03.pdf 
los mapuches, por ejemplo, la cultura del pueblo está íntimamente unida a la identidad religiosa. Se trata de una religiosidad vinculada con la tierra y la naturaleza, siendo una religión "cósmica, animista y chamánica": hay un poder divino, que está presente en una naturaleza animada y misteriosa, no siempre bondadosa, y son los chamanes los encargados de controlar esos poderes para restaurar el equilibrio ${ }^{45}$. Esto influye en el catolicismo al generarse una síntesis sincrética de diversas culturas, hay un enriquecimiento cultural en las tradiciones. Se trata de la religiosidad "expresada con los elementos culturales de que se dispone"46.

Así, al igual que ocurre con la lengua, en Iberoamérica encontramos una unidad religiosa con diferentes manifestaciones y matices.

\section{El rol de la cultura en las declaraciones de las Cumbres Iberoamericanas: análisis textual}

La cooperación cultural iberoamericana no es un invento actual: lleva ocurriendo ya desde el siglo XX. Las relaciones culturales comienzan a enriquecerse durante los años 20, época de la Generación del 98, momento en el que, metafórica y literalmente, Pablo Neruda y Federico García Lorca se conocen. Con la explosión de la Guerra Civil española y el triunfo del bando franquista se da el exilio republicano hacia Latinoamérica, una "fusión cultural" sobrevenida. Hay un hispanismo que forma parte de la política de Estado ya desde los años 40 y atraviesa toda la transición democrática, desembocando al final en el 92, con el lanzamiento de las Cumbres Iberoamericanas, en la búsqueda de una renovación de las relaciones iberoamericanas democráticas normalizadas. También cabe destacar la inmigración latinoamericana que se da en los años 80, otro intercambio cultural, pero esta vez a la inversa. Es decir, desde el punto de vista cultural ha habido diversas ocasiones de encuentro.

Entre las iniciativas actuales más importantes con respecto a la cultura Iberoamericana se encuentra la creación del concepto de Comunidad Iberoamericana de Naciones, cuyo primer germen se encuentra en la Organización de Estados Iberoamericanos (OEI), creada en 1949 como organismo de cooperación. Junto con la celebración de las primeras Cumbres Iberoamericanas de Jefes de Estado y de Gobierno, Iberoamérica comenzó a articularse. El principal elemento constitutivo se definió en la primera Cumbre Iberoamericana: el acervo cultural común ${ }^{47}$.

\footnotetext{
${ }^{45}$ https://web.uchile.cl/publicaciones/cyber/05/textos/riveros.html

46 Idelfonso Murillo Murillo, "La religión antes y después de las Independencias. ¿Fuente de unidad o de conflicto?”, Escritos, Vol. 19, N. 42, pp. 053-077, Medellín, Colombia, enero-junio 2011. http://www.scielo.org.co/pdf/esupb/v19n42/v19n42a03.pdf

${ }_{47}$ https://www.segib.org/cooperacion-iberoamericana/cultura/
} 
Las Cumbres Iberoamericanas de Jefes de Estado y de Gobierno surgieron gracias a una nueva mirada sobre las relaciones entre naciones, siendo un proyecto político que, consciente de las raíces históricas que vinculan a los países, toma esta característica para ahondar en nuevas formas de asociación en una época completamente diversa ${ }^{48}$. La diferencia se convierte en el nuevo elemento de unión: solo siendo conocedores de lo que une, puede profundizarse en lo que aporta diversidad y heterogeneidad para entrar en una nueva época de puesta en valor de lo propio de cada nación desde una mirada de horizontalidad.

El voluntarismo necesario en toda diplomacia se encuentra sin duda en la base de las relaciones Iberoamericanas. La Carta Cultural Iberoamericana (fruto de la cumbre iberoamericana celebrada en Montevideo, Uruguay, en el año 2006, junto con principios ya enunciados en la Declaración de la I Cumbre Iberoamericana celebrada en Guadalajara, México, en 1991), nace en un contexto en el que se destaca la utilidad de las relaciones internacionales iberoamericanas para reforzar la cultura común y a su vez revindicar la expresión plural existente. "La Carta Cultural es como una hoja de ruta que fue pionera a nivel mundial. Cuando la UNESCO estaba hablando de este tema Iberoamérica recogió el testigo e hizo una propuesta" ${ }^{\prime 4}$. De este modo el conjunto de naciones que forma Iberoamérica reconoce poseer un trasfondo compartido, con unas raíces y un patrimonio cultural fundados en la suma de pueblos y credos diversos.

Fruto de cada Cumbre encontramos también una Declaración firmada por los Jefes de Estado de los países representados. Por medio del análisis textual de las Declaraciones, buscamos observar de qué manera permea en la diplomacia iberoamericana, en las propias reuniones entre los Jefes de Estado, la idea de la centralidad de la cultura. Analizar las referencias a la cultura en las distintas declaraciones fruto de las Cumbres aporta una visión general del rol que ha ido adoptando. Hay que tener en cuenta que previo a la celebración de las Cumbres tienen lugar las Conferencias Iberoamericanas de Cultura, organizadas por la OEI, cuyos compromisos son incorporados a menudo a las Declaraciones de las Cumbres ${ }^{50}$.

\subsection{Década 1990: Identidad cultural}

A raíz de la observación de los usos y sentidos que se otorgan a la palabra cultura en la década de 1990, encontramos una imperante visión de la cultura desde el punto de vista antropológico, en especial en la concepción de la cultura como identidad (siendo "identidad cultural" el término que aparece en mayor

\footnotetext{
${ }^{48} \mathrm{https}$ //www.segib.org/las-cumbres-iberoamericanas-ahora-mas-que-nunca/

49 Arancha Álvarez, DG de Cultura de la OEI, en entrevista con Rut Sánchez Espiga el día 30/04/2020 vía Skype desde Madrid, archivada en soporte electrónico.

${ }^{50} \mathrm{https}$ //www.oei.es/historico/cic.htm
} 
número de textos, un total de 6 veces), como legado y como base del desarrollo. Destaca también que se emplee el término "cultura de paz", relacionando la cultura con la consecución de una paz duradera, y de "cultura de la innovación", que pone atención al modo en que la globalización puede ser beneficiosa para el intercambio.

Algunos elementos que ameritan atención son:

1. Se habla mucho de diversidad cultural pero no hay ninguna referencia a lo que une exactamente. Hay una falta de definición del término "acervo cultural compartido", y aunque se hace una continua alusión al valor de la lengua, no se hace mención directa a la raíz católica de los pueblos. Se habla de "una concepción común del ser humano y de su futuro".

2. La alusión a la "herencia cultural" de cada sociedad para justificar la falta de una "estrategia de desarrollo uniforme y universal". No se trata de homogeneizar las políticas, puesto que se debe tener en cuenta la diversidad de cada pueblo, pero debe ponerse suma atención en no justificar la desigualdad con un halo de "multiculturalismo".

3. La referencia ocasional a la cultura como instrumento y no "fundamento" del desarrollo, puesta al mismo nivel que las iniciativas en educación, salud, ciencia y tecnología, y no como elemento que debe subyacer a todas ellas.

4. En la IV Cumbre Iberoamericana, celebrada en Colombia y cuyo tema principal era económico, la cultura queda desplazada a un lado. Esto es una señal de alerta. Ocurre también en la IX Cumbre celebrada en Cuba que trata la situación financiera internacional en el ámbito de la globalización.

\subsection{Década 2000: Diversidad cultural}

En el año 2000 se celebra el Año Internacional de la Cultura de Paz. En el análisis de los usos de la palabra cultura en esta década, encontramos un gran énfasis en las acepciones en relación a la diversidad cultural y al diálogo entre culturas, a la interculturalidad. Es llamativo que ya no se utilice el término "identidad cultural". El acervo compartido es mencionado al comienzo de la gran mayoría de las Declaraciones de un modo automático, pero no se observa después un énfasis reflejado en el contenido de las mismas. Sí hay una mayor referencia al ámbito de la educación de la juventud y al tema de las migraciones, y en contraposición a los elementos de sociedad líquida de la década anterior, destaca que se haga alusión a la necesidad de transformar ciertos aspectos de las sociedades para el desarrollo de los pueblos. También es 
interesante el incremento en propuestas audiovisuales y la alusión al turismo como herramienta promotora de paz. Aun así, es llamativo que no haya un aumento en el uso del término "cultura de paz", dado el año.

Se observa en este periodo:

1. La todavía falta de definición del término "acervo cultural compartido" y su modestia en el uso del mismo.

2. La insistencia en la educación en una cultura de la "innovación" relacionada únicamente con lo científico.

3. La falta de concreción en relación a los "saberes ancestrales".

4. La alusión al modo en que las desigualdades educativas deben reducirse teniendo en cuenta la diversidad cultural. Si bien es cierto que es importante considerar las particularidades de cada cultura, en el ámbito de la desigualdad en la educación puede caerse en lo "políticamente correcto". Que las niñas no puedan estudiar, por ejemplo, no debe camuflarse bajo el "multiculturalismo".

\subsection{Década 2010: Bienes y servicios culturales, patrimonio cultural}

La cultura en la última década de las Cumbres adopta un claro sentido material, donde los términos asociados a la cultura que aparecen en mayor número de textos son "bienes y servicios culturales" y "patrimonio cultural", seguido por "industrias culturales". Esto muestra una clara tendencia materialista en la visión del significado de la cultura. En numerosas Declaraciones se hace hincapié en la necesidad de encontrar formas de medir el impacto de la cultura en la economía y se habla de la cultura en términos tangibles, donde aquello que no es medido pierde relevancia.

Además, todas las Cumbres llevan lemas asociados a la novedad, hablando de "transformación", "relación renovada", "nuevo contexto mundial", "innovación", "emprendimiento" y "prosperidad". La juventud cobra especial importancia, propiciando la movilidad académica, y los proyectos comienzan a incluir iniciativas que relacionan la cultura con el medio ambiente y la igualdad entre varón y mujer.

Podemos observar:

1. El término "acervo cultural" va muy a menudo vinculado a las industrias culturales. No termina de estar bien definido y aunque hace alusión a la lengua también se ve diluido entre conceptos de "interculturalidad" y "multiculturalidad".

2. La necesidad de medir "las potencialidades relacionadas al patrimonio cultural inmaterial", es decir, de calcular todo lo relacionado con la cultura. 
3. La focalización en la "cultura jurídica" compartida para resolver conflictos comerciales, sin hablar de valores compartidos y como estos podrían resolver además otro tipo de conflictos.

\section{La centralidad de la cultura: una diplomacia iberoamericana para el siglo XXI}

Si se prescinde de la cultura, de los símbolos y valores compartidos, la relación puramente económica se revela como un vínculo muy débil, ya que "se imponen con fuerza los intereses que, desde el punto de vista de los países latinoamericanos, pueden gestionarse mejor en términos bilaterales que propiamente iberoamericanos" $"$. Las relaciones conducidas dentro del espectro Iberoamericano solo tienen sentido si se ven sustentadas por un estrato común y, lo que es más, si se promociona esa base compartida como punto de partida para el crecimiento. Si se tratase de una unión puramente económica, los latinoamericanos, cada vez con mayor poder en el espectro internacional, fácilmente perderían el interés con respecto a España y Portugal, puesto que pueden manejarse en términos bilaterales dentro del propio continente. Si no se protege el componente ibérico de la cultura latinoamericana, la hegemonía cultural norteamericana y la incorporación de la mirada indígena pueden hacer olvidar los vínculos de hermandad que unen a Iberoamérica. Para poder llevar a cabo una diplomacia iberoamericana en el silgo XXI es necesario volver a lo básico, a las raíces que nos unen y nos permiten comprender dónde se encuentran nuestras cosmovisiones. Poner la cultura en el centro permitirá hacer renacer los lazos de unión que poseemos e iluminar el camino para la conducción de unas relaciones cada vez más estrechas y verdaderas.

\section{Conclusiones}

Por medio de la cultura el ser humano es capaz de relacionarse con la realidad, con el otro y consigo mismo. Se trata de la mirada a través de la cual comprende el mundo y por medio de la cual busca el sentido de la existencia. Si lo que diferencia a los pueblos es su diferente modo de abordar la realidad, el diálogo se encarga de establecer un puente de unión capaz de vincular a las sociedades por medio del encuentro de una base compartida. Por esto, el vínculo entre diplomacia y cultura es mucho más estrecho de lo que se ha solido advertir.

\footnotetext{
${ }^{51}$ Celestino Arenal, "Brasil, las Cumbres Iberoamericanas y el papel de España en América Latina”, CIDOB, Barcelona Centre For International Affairs, número 39, 2013.
} 
En nuestra propuesta para propiciar la paz, la denominada "diplomacia cultural" deberá hallarse en la base de toda política exterior y no ser considerada una mera forma de articular las relaciones, sino la base subyacente a todas ellas.

En el caso de las relaciones iberoamericanas, el concepto de cultura se ha ido devaluando. En las Declaraciones fruto de las Cumbres se observa una tendencia a percibir de forma materialista la cultura, acentuada en la última década. La comunidad Iberoamericana debe poner mayor énfasis en el acervo cultural compartido, concibiéndolo como una gran oportunidad de paz, definiéndolo con claridad y comprendiendo la dimensión inmaterial del mismo.

Finalmente, cabe concluir que en la cultura encontramos un modo de relación favorecedor del camino hacia la paz duradera entre los pueblos. Esto no significa que la búsqueda no vaya a ser, en muchas ocasiones, ardua -el bien es arduo- y no elimina la siempre presente posibilidad de la guerra, pero ciertamente ofrece un modo de vinculación permanente, puesto que conduce a crear lazos mucho más sólidos y estables.

\section{Referencias bibliográficas:}

Aníbal Heredia, Edmundo. "Relaciones internacionales latinoamericanas: historiografías y teorías", Estudios Ibero-Americanos, PUCRS, v. XXXIV, n. 1, 2008.

Arenal, Celestino, "Brasil, las Cumbres Iberoamericanas y el papel de España en América Latina", CIDOB, Barcelona Centre For International Affairs, número 39, 2013.

Bauman, Zygmunt. La cultura en el mundo de la modernidad líquida, Polity Press, 2011, p.75.

Calduch, Rafael. Dinámica de la Sociedad Internacional, Edit. CEURA. Madrid, 1993.

Dallmayr, Fred. "Dialogue among Civilizations: Some Exemplary Voices". Palgrave. Nueva York, 2002. en Eyzaguirre, Jaime. Hispanoamérica del dolor, Editorial Universitaria, Santiago de Chile, 1968.

Fernández Ochoa, José María, entrevista concedida a Rut Sánchez Espiga el día 27/04/2020 vía Skype desde Madrid.

Fernández Santamaría, María del Rosario. "Cultura y mundialización en el contexto iberoamericano", Pensar Iberoamérica, Revista de Cultura, Organización de Estados Iberoamericanos.

Ferreira Gomes, Nancy Elena. España y Portugal en el espacio Iberoamericano: coincidencia en las prioridades de política externa, Real Instituto Elcano, 2017.

Figueiras Fernández-Ojanguren, Alfredo. Ciudades Españolas en América, Ediciones Lacre, 2017.

Iglesias, Enrique. "El español en iberoamérica: lengua e identidad", TELOS 71: El valor económico del español en la Sociedad Global, Agenda, $\mathrm{n}^{\circ} 71$.

Frago Gracia, Juan Antonio. Entre América y España, el vínculo de la lengua, Boletín de Filología vol.47 no.2 Santiago dic. 2012. 
Giraldo, Manuel Lucena, entrevista concedida a Rut Sánchez Espiga el día 06/05/2020 vía video llamada desde Madrid.

Isaac Caro, Isabel Rodríguez, "El enfoque del diálogo civilizacional desde América Latina", Revista de Relaciones Internacionales, Estrategia y Seguridad, vol. 11, núm. 1, Bogotá, Colombia, 2016.

Macionis, John, Plummer, Ken. Sociología, Prentice Hall, 1999.

Mansilla, H.C.F."La influencia de elementos religiosos sobre la cultura política en América Latina", en Fragmentos de Filosofia, no 10 (2012), pp. 91-115.

Marichalar, Carlos. Algunas reflexiones sobre la historia de los intelectuales/diplomáticos latinoamericanos en los siglos XIX y XX, Revista de Historia de América, Instituto Panamericano de Geografía e Historia, núm. 156, 2019.

Ministerio de Relaciones Exteriores de Colombia y la UNESCO (Bogotá 27 y 28 septiembre 2007).

Montiel, Edgar, Intervención en el Encuentro andino sobre Diplomacia Cultural. Seminario convocado por el Intervención en el Encuentro andino sobre Diplomacia Cultural. Seminario convocado por el Ministerio de Relaciones Exteriores de Colombia y la UNESCO (Bogotá 27 y 28 septiembre 2007).

Murillo Murillo, Idelfonso. "La religión antes y después de las Independencias. ¿Fuente de unidad o de conflicto?", Escritos, Vol. 19, N. 42, pp. 053-077, Medellín, Colombia, enero-junio 2011.

Nelson, James M. Psychology, religion and spirituality, Springer Science \& Business Media, 2009.

Neruda, Pablo. "Las palabras". Disponible en Revista Altazor, Revista Electrónica de Literatura, año 3, enero 2021. https://www.revistaaltazor.cl/pablo-neruda-laspalabras/.

Parra, Nicanor, Discursos de sobremesa, Ediciones Universidad Diego Portales, Santiago de Chile, 2006.

Posner, R."Basic tasks of cultural semiotics", en: G. Withalm \& J. Wallmannsberger (Eds.). Signs of power - power of signs. Essays in Honor of Jeff Bernard. Vienna: INST.p. 10. (pp.56-89).

Ramírez Calzadilla, Jorge. "La religiosidad americana y caribeña. Un elemento de la identidad cultural", en América Latina y El Caribe. Realidades Sociopolíticas e Identidad Cultural, Ediciones Heinrich Böll, El Salvador, 2002, pp. 215-236. Disponible en http://biblioteca.clacso.edu.ar/ar/libros/cuba/cips/caudales05/ Caudales/ARTICULOS/ArticulosPDF/0915R072.pdf.

Vilariño Pintos, Eduardo. Curso de Derecho Diplomático y Consular. Parte General y textos codificados, Editorial Tecnos, Madrid, 1987.

Zerba De Crispis, Yubeira. "Un acercamiento al fenómeno religioso en América Latina a la luz de la crítica cultural”, en Sapienza Organizacional, vol. 5, núm. 9, 2018, disponible en https:/www.redalyc.org/jatsRepo/5530/553056570011/html/index. $\underline{\mathrm{html}}$ 


\section{Fuentes personales:}

Álvarez, Arancha, DG de Cultura de la OEI. Entrevista realizada el 30/04/2020 vía videollamada.

González Ochoa, José María, Investigador especializado en la época de la Conquista y coordinador de proyectos para SM España y América Latina. Entrevista realizada el 27/04/2020 vía videollamada.

Lucena Giraldo, Manuel, Historiador doctorado en Historia de América, investigador del CSIC, profesor, experto en educación y cultura. Entrevista realizada el 06/05/2020 vía videollamada.

Málaga Shaw, Paloma, Coordinadora de la gestión Cultural del Área de Educación del Museo del Prado. Entrevista realizada el 27/04/2020 vía email.

Read Escobal,Virginia, creadora Fundación Cultural "Lleva un libro en la maleta", que conecta España con la República Dominicana. Entrevista realizada el 22/04/2020 vía telefónica.

Consulta web [22/05/2020]:

Asociación de ACAdEmias de la Lengua Española. URL:https://www.asale.org/sites/ default/files/Folleto de ASALE.pdf

Aznárez, Juan Jesús, Vicent, Mauricio, “Cuba denuncia la injerencia 'vergonzosa' de Estados Unidos en la Cumbre de la Habana”, El País, noviembre 1999.

URL:https://elpais.com/diario/1999/11/14/internacional/942534004_850215.html

Centro Internacional de Cultura de Paz.

URL: https://www.organismointernacional.org/cultura- de-paz.php

COMISIÓN ECONÓMICA PARA AmÉRICA LATINA Y EL CARIBE.

URL:https://celade.cepal.org/redatam/PRYESP/SISPPI/Webhelp/porcentaje de indigenas_habla_idi oma_indigena_y_castellano.htm

Dastis, Alfonso, "El compromiso de España con América Latina", ElEconomista,es, 2018.

URL: $\quad$ https://www.eleconomista.es/especial-america/noticias/9170918/05/18/Elcompromiso- de-Espana-con-America-Latina.html

Grynspan, Rebeca, "Las Cumbres Iberoamericanas, ahora más que nunca", Le Monde Diplomatique en español, 2018. URL: https://mondiplo.com/las-cumbresiberoamericanas- ahora-mas-que-nunca

Iglesias, Enrique, "En 25 años casi todo un siglo", EL PAIS, 2016. URL:https://elpais.com/ elpais/2016/07/13/opinion/1468432062_049818.html

Instituto Cervantes, “La institución”. URL:

https://www.cervantes.es/sobre instituto cervantes/informacion.htm

Ministerio de Asuntos Exteriores, Unión Europea y Cooperación, Gobierno de España, "España en Iberoamérica".

URL:http://www.exteriores.gob.es/Portal/es/PoliticaExteriorCooperacion/Iberoamerica/ Paginas/EspE nIberoamerica.aspx

Quilodrán, Federico, "Hoy se reúnen los jefes de estado en la VI Cumbre Iberoamericana", La Nación, 1996. URL: https://www.lanacion.com.ar/politica/hoy-se-reunen-losjefes-de- estado-en-la-vi-cumbre-iberoamericana-nid169902/

Riveros, María Elena, "Religión e identidad en el pueblo Mapuche", CYBER HUMANITATIS, n5. 1998.

URL:https://web.uchile.cl/publicaciones/cyber/05/textos/riveros.html

Secretaría General Iberoamericana, "Cultura".

URL:https://www.segib.org/cooperacion- iberoamericana/cultura/ 


\section{Fuentes audiovisuales:}

Roca Barea, María Elvira. Intervención en "Mesa redonda: EEUU, España y el mundo hispánico. Una mirada al futuro".

URL:https://www.youtube.com/watch?v=njI81X4E0IQ

\section{Documentos Cumbres Iberoamericanas:}

Declaración de Guadalajara. México. 19 de julio de 1991.

Declaración de Madrid. España. 23 y 24 de julio de 1992.

Declaración de Salvador, Brasil. 15 y 16 de julio de 1993.

Declaración de Cartagena, Colombia. 14 de julio de 1994.

Declaración de Bariloche, Argentina. 16 y 17 de octubre de 1995.

Declaración de Viña del Mar, Chile. 10 y 11 de noviembre 1996.

Declaración de Isla de Margarita, Venezuela. 8 y 9 de noviembre 1997.

Declaración de Oporto, Portugal. 17 y 18 de octubre 1998.

Declaración de La Habana, Cuba. 15 y 16 de noviembre de 1999.

Declaración de Lima. Perú. 23 y 24 de noviembre de 2001.

Declaración de Bábaro. República Dominicana. 15 y 16 de noviembre de 2002.

Declaración de Santa Cruz de la Sierra. Bolivia. 14 y 15 de noviembre de 2003.

Declaración de San José. Costa Rica. 18 y 20 de noviembre de 2004.

Declaración de Salamanca. España. 14 y 15 de octubre de 2005.

Declaración de Montevideo. Uruguay. 3, 4, 5 de noviembre de 2006.

Declaración de Santiago. Chile.8, 9,10 de noviembre de 2007.

Declaración de San Salvador. El Salvador, 29 a 31 de octubre, 2008.

Declaración de Lisboa. Portugal. 30 de noviembre y 1 de diciembre de 2009.

Declaración de Mar de Plata. Argentina, 2010.

Declaración de Asunción. Paraguay. 28 y 29 de octubre de 2011.

Declaración de Cádiz. España. 16 y 17 de noviembre de 2012.

Declaración de Panamá. Panamá. 18 y 19 de octubre de 2013.

Declaración de Veracruz. México. 8 y 9 de diciembre de 2014.

Declaración de Cartagena de Indias. Colombia. 28 y 29 de octubre de 2016.

Declaración de Antigua Guatemala. Guatemala. 15 y 16 de noviembre de 2018. 
